\title{
Haití, la nueva comunidad inmigrante en Chile
}

\author{
Haiti, new immigrant community in Chile
}

\author{
Katherin Sánchez P.a, Jaime Valderas J., \\ Carolina Sánchez G.a, ${ }^{\mathrm{a},}$, Francisco Barrera Q.,
}

\author{
aUnidad de Agudos, Servicio de Pediatría Hospital Clínico San Borja Arriarán \\ ${ }^{\text {b} A c a d e ́ m i c o ~ D e p a r t a m e n t o ~ d e ~ P e d i a t r i ́ a ~ C e n t r o . ~ U n i v e r s i d a d ~ d e ~ C h i l e ~}$ \\ cProfesor Asociado de Pediatría Universidad de Chile
}

Recibido el 04 de noviembre de 2017, aceptado el 27 de febrero de 2018

\begin{abstract}
Resumen
Las migraciones constituyen un fenómeno creciente en América Latina, influido por factores económicos, laborales, búsqueda de bienestar social, educación y salud, entre otros. Chile presenta una tasa neta de migración positiva, y en el último tiempo ha destacado el gran número de inmigrantes haitianos que han llegado a nuestro país, especialmente luego del terremoto que le afectó el 2010. Provenientes de una cultura diferente, donde el estilo de crianza y las características de atención en salud, entre otros aspectos, difieren a la de nuestro país, conocer la cultura haitiana y su situación de salud es relevante para una mejor apreciación de sus necesidades y enfrentar de mejor forma la programación de la atención sanitaria de esta población que busca en Chile un lugar de acogida y mayor bienestar. Para profundizar los esfuerzos de integración en trabajo, salud, educación y en la comunidad parece aconsejable actualizar la legislación referente a migraciones, tal que permita abordar los problemas actuales a través de una ley migratoria que data de 1975.
\end{abstract}

\begin{abstract}
Migration is a growing phenomenon in Latin America influenced by several factors such as economic stability, employment, social welfare, education and health system. Currently Chile has a positive migration flow rate. Particularly, a significant number of Haitian immigrants has been observed during the last years, especially after earthquake of 2010. These immigrants present a different cultural background expressed in relevant aspects of living including parenting and healthcare. Knowing the Haitian culture and its health situation is relevant for a better understanding of their health needs. Haitian people come to Chile looking for a cordial reception and willing to find a place with better perspectives of wellbeing in every sense. Immigration represents a major challenge for Chilean health system that must be embraced. Integration efforts in jobs, health, education system and community living should be enhanced to ensure a prosper settlement in our country. A new immigration law is crucial to solving major problems derived from current law created in 1975.
\end{abstract}

Palabras clave: Inmigración; Haití;

Creole; religión vudú; integración

\section{Keywords:} Immigration; Haiti; Creole; integration; voodoo religion 


\section{Inmigración y sus consecuencias}

Las migraciones constituyen un proceso creciente en América Latina (AL), fenómeno que se acompaña de un impacto en la comunidad en general y en particular en aspectos epidemiológicos en salud, especialmente en el ámbito de salud materno infantil ${ }^{1,2}$. A diferencia de siglos precedentes, hoy se aprecia una mayor tasa neta de migración (TNM) entre los países en vías de desarrollo de AL, indicador que hasta ahora es positivo para Costa Rica, Panamá, Venezuela, Chile y Argentina ${ }^{1-3}$. Los movimientos migratorios, están influidos por factores económicos, laborales, búsqueda de bienestar social, de mejores condiciones de educación y salud, desplazamientos por violencia, terrorismo e inseguridad y mejores perspectivas de desarrollo profesional ${ }^{3,4}$. Estos condicionantes deben considerarse al estudiar este proceso social ya que, además de beneficios para el país de acogida, pueden ocasionar o favorecer modificaciones en indicadores de salud y epidemiología local ${ }^{4,5}$.

En la época colonial, la inmigración de personas afrodescendientes fue escasa y circunscrita al norte del país, constituyendo aún en la región un pequeño grupo de descendientes ${ }^{4,6}$. La estabilidad económica comparativa de Chile en los últimos años, la disminución sostenida de la tasa de fertilidad y la presencia de conflictos internos y desastres naturales que han afectado otros países de AL, han generado condiciones que han determinado, desde 1995, nuevas olas de inmigrantes ${ }^{1,3}$. De acuerdo a estudios de Amnistía Internacional, el Departamento de Economía y Asuntos Sociales de la ONU y la Organización Internacional para la Migración (IOM), desde 1980 hasta el 2017 el número de inmigrantes en Chile se quintuplicó, alcanzando los 488.600 habitantes. (https://migrationdataportal. org/?i=stock_abs_\&t $=2017 \& \mathrm{~cm} 49=152$, consultada en febrero 2018). Por su parte, el Censo 2017 permitió estimar que la población total de inmigrantes en ese año alcanzaría las 600.000 personas, lo que representa el $2,7 \%$ de la población total ${ }^{7,8}$. Estimaciones del Departamento de Migración y Extranjería de Chile (DEM) señalan que Haití ocupa el $5^{\circ}$ lugar con $4,1 \%$ de los inmigrantes, principalmente afrodescendientes, y que del total de inmigrantes con permanencia definitiva al año 2016 en la Región Metropolitana, quienes provienen de Haití y Venezuela constituyen, respectivamente, un $10,6 \%$ y $9,6 \%$, convirtiéndose en la tercera y cuarta colonias más grandes de la capital, sólo después de peruanos $(30,8 \%)$ y colombianos $(18,9 \%)^{8}$.

A diferencia de la inmigración europea de fines del siglo XIX y principios del siglo XX, que contaba con un marco regulatorio y un sistema de apoyo estructurado, en las corrientes migratorias actuales se percibe cierta tensión, originada muchas veces en vulneracio- nes de los derechos de los migrantes. Organismos internacionales han constatado deficiencias significativas en materia de protección de derechos de las personas inmigrantes en Chile, concluyendo la necesidad de revisar íntegramente la legislación del estatuto jurídico del inmigrante $e^{1,2,6}$.

\section{Haití}

La Española o isla de Santo Domingo fue el primer asentamiento europeo en el Nuevo Mundo. Forma parte de las Grandes Antillas y acoge a dos estados soberanos: Haití y República Dominicana. Haití fue el primer país de AL en lograr su independencia y abolir la esclavitud. Su lengua nativa es el creole, pero por colonización adquirió el francés ${ }^{9,10}$.

Posee 10.911.819 habitantes, $60 \%$ de raza negra, $30 \%$ de mulatos y menos del $10 \%$ de raza blanca, principalmente de ascendencia francesa. Corresponde a la nación más pobre de América y del hemisferio oeste, con un PIB per cápita del año 2016 de US\$ 820, una de las menores expectativas de esperanza de vida: 64 años y una tasa de alfabetización de las más bajas del mundo: $76 \%$, lo que lo clasifica como nación con bajo desarrollo humano según el PNUD ${ }^{11}$. Su tasa de fertilidad para el año 2015 fue 2,79 y la tasa de natalidad de 23,3 comparada con las de 1,7 y 13,05 de Chile, respectivamente ${ }^{9}$. De religión católica predominante, existe una coexistencia y fusión con la práctica del vudú. Su evolución como nación se ha caracterizado por conflictos internos recurrentes como revoluciones, golpes de estado y dictaduras ${ }^{10}$.

La estructura social es de tipo patriarcal, aunque ha habido un progresivo mayor rol de la mujer. Ella desempeña el rol de ama de casa y se la ve como "poto mitan" o pilar central de la familia. La red familiar en Haití sigue el concepto de familia/pueblo y su núcleo familiar primario abarca los círculos tradicionales de primero, segundo y tercer grado ${ }^{12}$. La religión es importante para la sociedad ya que contribuye en impartir justicia y ofrecer soluciones curativas, teniendo influencia en el sistema educacional, en especial las instituciones católicas. En su tradición, la narración de cuentos de origen africano, la música, sus bailes, la tradicional sopa de zapallo y una veneración a lo sobrenatural son fundamentales como expresión de identidad nacional ${ }^{10}$.

\section{La salud en Haití}

El sismo del año 2010 generó el "Plan de acción para la recuperación y el desarrollo de Haitî", que incluyó acciones específicas en salud. Posteriormente, el 
año 2015, se definió un marco sanitario nuevo, especialmente en lo referente a la calidad del agua destinada al consumo humano, privilegiando el saneamiento, la higiene y la descontaminación de las fuentes ${ }^{13}$.

Su sistema de salud está constituido por prestadores del sector público $(35,7 \%)$, privado $(32,5 \%)$, mixto no lucrativo $(31,8 \%)$ y el sector tradicional. El sector público está regulado por el Ministerio de Salud Pública y al año 2016 había 1,4 médicos y 1,8 enfermeras por 10.000 habitantes, lejos de la norma mínima de la OMS de 25 profesionales por cada 10.000 habitantes $^{9-11}$. Estos índices han mejorado, contribuyendo a ello acuerdos de cooperación con varios países de América Latina y el Caribe (ALC). El sector privado está constituido por profesionales que trabajan por cuenta propia o en clínicas. Casi el $80 \%$ de toda la población tiene acceso a las prácticas de una medicina complementaria ampliamente extendida, en tanto que la medicina moderna con frecuencia es el último recurso frente a una enfermedad, especialmente para quienes viven en sectores rurales, dependiendo de su educación y de sus convicciones religiosas ${ }^{10,11}$.

La malaria es endémica y Haití concentra el mayor número de casos en ALC (17.696 casos el año 2014), que ha sido difícil de erradicar por las propias condiciones de salud y geografía ${ }^{13,14}$.

La prevalencia de tuberculosis (TBC) no es fácil de estimar ya que organizaciones externas financian los programas de tratamiento. Al año 2015 la OMS estimó una prevalencia de 250 por 100.000 h, con un éxito terapéutico de $80 \%$ en el grupo virgen a tratamiento. La TBC resistente a drogas en los casos nuevos alcanza a un 3\%. Respecto a VIH, los datos obtenidos son aún más escasos, estimando la OMS al año 2013, una incidencia de 65 casos por $100.000 \mathrm{~h}$ y una prevalencia de 1.346 casos por $100.000 \mathrm{~h}$, con elevada mortalidad ${ }^{15}$.

La anemia es frecuente. En anemias importantes, dada la constitución genómica de afrodescendencia, debe considerarse la anemia de células falciformes y la talasemia, cuyas crisis pueden confundirse con una infección, como causa o factor de la descompensación, que puede tener manifestaciones clínicas muy graves secundarias el síndrome vaso oclusivo, requiriendo una sospecha diagnóstica oportuna ${ }^{16}$.

\section{Salud materno infantil en Haití}

La salud materna ha progresado significativamente en Haití, aunque las cifras al año 2015 son las de mayor riesgo de la región. La atención profesional del parto alcanza solo el 50\%, con una mortalidad materna que ha descendido desde 630 muertes por cada 100.000 nacidos vivos (NV) en el período 2005-2006, a 359 muertes por $100.000 \mathrm{NV}$ el año $2013^{11,13,17}$, aun- que las cifras oficiales del país señalan 157 muertes por $100.000 \mathrm{NV}^{13}$. Según la ONU, una mujer en Haití tiene una probabilidad de 1 en 80 de morir debido a un embarazo o parto, en comparación con el riesgo regional de uno en $510^{11}$. En la población rural muchas mujeres no asisten a control médico durante su embarazo, pero suelen acudir a una "femme sage" (partera) utilizando medicina complementaria ${ }^{10}$. La tasa de mortalidad infantil desciende de 73,45 por 1.000 recién nacidos vivos (RNV) el 2005 a 59 x 1.000 RNV el 2013, aunque continúa siendo la más alta del continente ${ }^{17}$.

\section{Migración a Chile}

Los bajos niveles de educación y la dinámica familiar patriarcal ya señalada, expone al varón a aceptar ofertas de trabajo mal remunerado y a la búsqueda de vivienda en sectores socialmente vulnerables, a veces en condiciones de hacinamiento. La población inmigrante y su cultura repercuten en el barrio e impactan en la calidad de vida de la comunidad, surgiendo una nueva expresión de vulnerabilidad urbana ${ }^{1,3,11}$. Sin embargo, las condiciones de vida de los inmigrantes en nuestro país son menos precarias que en otros países de $\mathrm{AL}^{3}$. Las limitaciones en la comunicación verbal contribuyen a dificultar una mejor calidad de vida y de relación con su entorno; adicionalmente, las costumbres sociales suelen chocar con nuestros códigos culturales, dificultando la integración social ${ }^{5,11}$. Frecuentemente se produce una inmigración primaria del padre de familia, que establece redes laborales y sociales, avanza en el manejo del idioma y se establece muchas veces el concepto de "economía étnica", haciendo referencia a la utilización de recursos compartidos entre pares y al desarrollo de pequeños emprendimientos propios de sus costumbres y competencias ${ }^{3}$.

Los inmigrantes haitianos se han establecido preferentemente en el Gran Santiago, en comunas como Quilicura, Independencia, Estación Central y Santiago Centro, ampliándose en el último tiempo a provincias. Es la migración que más ha crecido en Chile ${ }^{1,18}$. En el Censo de 2002 los haitianos residentes en Chile eran sólo 50 personas, observándose un incremento de $731 \%$ entre 2013 y 2016, período en que ingresaron 41.000 personas $^{19}$. Durante el año 2016, más de 10 mil haitianos llegaron al país asentándose en Quilicura, comuna que ha debido implementar un programa social para su integración ${ }^{3,18}$. El desafío global y multidimensional que impone este numeroso arribo es evidente $e^{1-3,5}$.

La creación del Consejo Técnico de Política Migratoria el año 2015 ha significado un importante avance en la regularización e integración de los inmigrantes de diversas nacionalidades a nuestro país ${ }^{1,19}$. Los países 
integrantes de la Organización para la Cooperación y Desarrollo Económico (OCDE) recomiendan un porcentaje de inmigración de $4 \%$ de la población, existiendo aún en nuestro país una brecha del $30 \%$ para esta meta $^{11,13}$, lo que hace imprescindible actualizar nuestra legislación al respecto.

\section{Salud Materno Infantil de las inmigrantes haitianas en Chile; experiencia del Hospital Clínico San Borja Arriarán (HCSBA)}

En Chile, en el ámbito de la salud de la embarazada inmigrante, los mecanismos sociales de protección a la madre y su futuro hijo fueron en sus inicios escasamente considerados, lo que se reflejó en la caída de indicadores tradicionalmente muy promisorios como los de mortalidad materna, mortalidad fetal, y aquellos relacionados a lactancia que permiten acreditar como hospital amigo de la madre y el niño $0^{5,17}$. En relación a nacimientos, datos del Registro Civil señalan que el año 2010, solo un 3,3\%, de los nacidos en Chile tenía al menos un padre extranjero; al año 2017, dicha cifra se ha elevado a 8,6\% (18.822 RN de un total de 219.429 nacimientos) $)^{17,19}$.

Nuestro país al año 2013, ocupaba el tercer lugar de América en el menor índice de mortalidad materna con 22 por $100.000 \mathrm{NV}$, solo superado por Canadá (11 por $100.00 \mathrm{NV}$ ) y Uruguay (14 por $100.000 \mathrm{NV}$ ). Le seguía en cuarto lugar EE. UU. con 28 x $100.000 \mathrm{NV}$, en tanto que Haití presentaba la tasa más alta con 359 $\mathrm{x} 100.000 \mathrm{NV}^{17}$. El porcentaje de partos en población haitiana en el año 2016 en el HCSBA fue de 7.5\%. El año 2017, el porcentaje total de partos de mujeres inmigrantes alcanzó el 60\%, siendo especialmente notorio el incremento de partos de mujeres provenientes de Haití, alcanzando la segunda nacionalidad en partos de inmigrantes, después de Perú, con un $26.5 \%$ del total de partos ${ }^{20}$. En el HCSBA la mortalidad materna en el año 2016 aumentó significativamente a 70.83 por $100.000 \mathrm{NV}^{20}$ debiéndose lamentar tres muertes maternas ese año, todas inmigrantes, dos de ellas haitianas. Según el informe del Comité de auditoría del HCSBA, en la embarazada inmigrante se ha observado falta de control del embarazo y pesquisa tardía de morbilidad materna y fetal ${ }^{20}$. Las dificultades para establecer los controles se originan en falta de disponibilidad de la documentación necesaria, desconocimiento del sistema oficial de salud, escasa disponibilidad de redes de apoyo social, etc. Esto dificulta o impide la pesquisa oportuna de condiciones de riesgo, del seguimiento de embarazos de alto riesgo como síndrome hipertensivo del embarazo o diabetes y no se alcanza a cumplir el protocolo de ecografías prenatales ${ }^{17,20}$.

La mortalidad fetal en el HCSBA, aumentó a 10.3 por mil en el año 2016, 44 mortinatos de un total de 4.297 nacimientos $^{20}$. Dicha cifra corresponde a un aumento de casi 3 veces la tasa nacional que se había mantenido estable en cifras de 4 por mil nacidos vivos (INE 2015).

El problema más grave es la barrera idiomática, que dificulta la comprensión de instrucciones, incluso las básicas. El HCSBA dispone de algunas funcionarias haitianas contratada para facilitar la comunicación en su lengua nativa y se dispone de información e instructivos en español y creole en la red del SSMC. Algunas trabas administrativas que suelen retrasar la atención de embarazadas que requieren asistencia urgente y la ausencia de documentación que acredite identidad (pasaporte, DNI, CI) que puede retrasar el alta del recién nacido son otros ejemplos de dificultades que afectan la atención de salud ${ }^{5,19}$. Adicionalmente, las precarias condiciones de vivienda, problema creciente, genera trabas para el alta segura de la diada madrehijo, especialmente del recién nacido, lo que se traduce en mayor permanencia hospitalaria ${ }^{19,20}$.

En el caso de recién nacidos con necesidad de cama crítica, se ha visto dificultado el inicio y la mantención de la lactancia, ante la actitud fatalista familiar que anticipa un duelo y determina conductas de resignación y abandono, deteriorando indicadores de lactancia al alta según apreciación de las matronas de Puericultura y equipo médico de nutriólogos del HCSBA ${ }^{20}$. Según informe UNICEF del año 2015, en Haití se observa un $39,7 \%$ de lactancia materna exclusiva a los 6 meses $^{13}$.

En morbilidad pediátrica, suele observarse consulta tardía en los servicios de urgencia, incumplimiento de las indicaciones médicas, y dificultades en el proceso diagnóstico secundario a las trabas presentes en la comunicación verbal ${ }^{5}$. Dada su cultura de escaso acceso a atención en salud en su país de proveniencia, los servicios médicos obstétricos, neonatológicos y pediátricos en Chile deben reevaluar la atención médica, optimizando el rol del nivel primario e introducir mejorías en la comunicación a través de intérpretes y/o traductores, cuya labor la pueden desempeñar los mismos ciudadanos haitianos que ya llevan algunos años en nuestro país ${ }^{12,18}$ como ya lo están desarrollando algunos gobiernos regionales y comunales, a través de programas específicos ${ }^{18}$.

Adicionalmente, se han desarrollado instancias específicas para la regularización de niños y adolescentes inmigrantes, como la creación de una visa especial que permitirá acceder a prestaciones de salud y beneficios educacionales cuyo único requisito será contar con el certificado de nacimiento legalizado ${ }^{19}$. Junto a los esfuerzos desplegados por diversos departamentos de los ministerios relacionados, como Extranjería y Migración, instituciones religiosas, diversas organizaciones no gubernamentales (ONG), existen instancias creadas 
por los mismos inmigrantes, con el propósito de avanzar en su mayor estabilización en nuestro país ${ }^{18}$.

\section{Conclusiones}

El impacto de las migraciones existe y se está en un proceso de favorecer los aspectos positivos como es el efecto sobre la reducida tasa de natalidad nacional, incorporación a la fuerza de trabajo y apertura a otras visiones de la vida. Así, aunque con diversos grados de dificultad, el proceso de integración se está llevando a cabo, observándose en forma creciente diversas instancias de integración y acogida que buscan facilitar el desempeño laboral ${ }^{21}$, contribuyendo significativamente a ver cumplidos anhelos y atenuando el denominado Sindrome de Ulises o del emigrante con estrés crónico y múltiple 22 .

Las migraciones son un fenómeno complejo y multivariable, con influencia decisiva y positiva en la historia y desarrollo de los países ${ }^{1,2}$. La situación de la inmigración en Chile necesita pasar de una inmigración libre, de fronteras casi abiertas, a una inmigración regulada a través de la esperada Ley de Migración ${ }^{19}$, lo que permitirá enfrentar mejor el impacto señalado.

\section{Responsabilidades éticas}

Protección de personas y animales: Los autores declaran que los procedimientos seguidos se conformaron a las normas éticas del comité de experimentación humana responsable y de acuerdo con la Asociación Médica Mundial y la Declaración de Helsinki.

Confidencialidad de los datos: Los autores declaran que han seguido los protocolos de su centro de trabajo sobre la publicación de datos de pacientes.

Derecho a la privacidad y consentimiento informado: Los autores han obtenido el consentimiento informado de los pacientes y/o sujetos referidos en el artículo. Este documento obra en poder del autor de correspondencia.

\section{Conflicto de intereses}

Los autores declaran no tener conflicto de intereses.

\section{Referencias}

1. Cabieses B, Bernales M, McIntyre A. La migración internacional como determinante social de la salud en Chile: evidencia y propuestas para políticas públicas. Programa de Estudios Sociales en Salud, Universidad del Desarrollo, 2017. http://www.udd.cl/dircom/pdfs/ Libro_La_migracion_internacional.pdf (consultada en febrero de 2018). ISBN versión digital: 978-956-374-001-1.

2. Bellolio A, Errázuriz H. Migraciones en Chile. Oportunidad ignorada. 1a Edición, Santiago de Chile 2014. Ediciones L y D.

3. Rojas N, Koechlin J (eds). Migración Haitiana hacia el Sur Andino. $1^{\text {a }}$ edición. Madrid 2017. Centro de Ética y Reflexión Social, Servicio Jesuita a Migrantes de Chile/Centro Fernando Vives, U. Alberto Hurtado de Chile/U. Antonio Ruiz de Montoya de Perú.

4. Tijoux M, Córdova M. Racismo en Chile: colonialismo, nacionalismo, capitalismo. Polis, Revista

Latinoamericana 2015;14(42):7-13.

5. Vásquez R. Impacto de las migraciones en Chile: Nuevos retos para el pediatra. ¿Estamos preparados? Rev chil pediatr. 2009;80(2):161-7.

6. Rojas N, Amode N, Vásquez J. Racismo y matrices de "inclusión" de la migración haitiana en Chile. Polis, Revista Latinoamericana 2015;14(42):21745 .
7. Ministerio de Economía. Resultados Censo 2017. http://www.censo2017.cl/wpcontent/uploads/2017/12/Presentacion Resultados_Definitivos_Censo2017.pdf (consultada en febrero de 2018).

8. Departamento de Extranjería y Migración, Chile (2016). Estadísticas de Migración. http://www.extranjeria.gob.cl/estadisticasmigratorias/ (consultada en febrero de 2018).

9. Central Intelligence Agency (CIA). Haiti: The world factbook, updated january 23, 2018. https://www.cia.gov/library/ publications/the-world-factbook/geos/ ha.html (consultada en febrero de 2018).

10. Brice Fundation International. Haití, Cultura y Tradiciones. http://www. bricefoundation.org/haitian-culture-andtradition (consultada en febrero de 2018).

11. Programa de las Naciones Unidas para el Desarrollo (PNUD). Informe sobre desarrollo humano 2016. http://hdr. undp.org/sites/default/files/HDR2016_ SP_Overview_Web.pdf (consultada en febrero de 2018).

12. Mertlet M. Haití: Mujeres en busca de la ciudadanía de pleno derecho en una transición sin fin. En: Lebon N, Naier E (ed.): De lo privado a lo público: 30 años de lucha de las mujeres en América Latina. 2016; Ed. Siglo XXI México.

13. Pan American Health Organization (PAHO). Salud en las Américas 2017. https://www.paho.org/saluden-las-americas-2017/?page_t_es.../ haiti\&lang=es (consultada en febrero de 2018).

14. Boncy PJ, Adrien P, Lemoine JF, et al. Malaria elimination in Haiti by the year 2020: an achievable goal? Malar J. 2015;14:237.

15. Programa de las Naciones Unidas para el Desarrollo (PNUD). Haití lucha contra el SIDA y la tuberculosis. 2017 http://www. latinamerica.undp.org/content/rblac/es/ home/ourwork/democratic-governance/ successstories/haiti_s-fight-against-aidsand-tuberculosis.html (consultada en febrero de 2018).

16. United States Agency for International Development (USAID). Haiti National Anemia Profile. https://www.springnutrition.org/sites/default/files/ publications/anemia-profiles/spring_nap_ haiti.pdf (consultada en febrero de 2018).

17. OMS y Gobierno de Chile. Mortalidad materna y Neonatal en América Latina y el Caribe (ALC). http:// www.who.int/pmnch/activities/ sintesis_situacionmortalidad_en_alc.pdf (consultada en febrero de 2018).

18. Margarite D, Bijit K. Barrios y población inmigrante. el caso de la comuna de Santiago. Revista INVI (on line) 2014; 29 (81):19-77. http://revistainvi.uchile. cl/index.php/INVI/article/view/831/1153 (consultada en febrero de 2018).

19. Ministerio del Interior, Depto. de Extranjería. Enero 2018: Consejo técnico de política migratoria realiza 
Inmigración en Chile - K. Sánchez P. et al

balance de acciones realizadas en dos años. http://www.extranjeria.gob.cl/ noticias/2018/01/05/ (consultada en febrero de 2018).

20. Ministerio de Salud, SSMC. Hospital San Borja Arriarán, Servicio de Pediatría.
Informes de auditoría materno infantil HCSBA años 2016-2017.

21. Santiagointegrado.cl. Instituciones de ayuda a migrantes http://www. santiagointegrado.cl/ayuda-a-migrantes (consultada en febrero de 2018).
22. Achotegui J. Estrés límite y salud mental: el síndrome del inmigrante con estrés crónico y múltiple (Síndrome de Ulises). Revista Norte de salud mental, Sociedad Española de Neuropsiquiatría 2005;5(21):39-53. 